Case Report

\title{
Pulmonary Nocardiosis in an Immunocompetent Patient with Cystic Fibrosis
}

\author{
Lucy Schoen, ${ }^{1}$ Jonathan D. Santoro, ${ }^{2}$ Carlos Milla, ${ }^{3}$ and Sumit Bhargava ${ }^{3}$ \\ ${ }^{1}$ Stanford University School of Medicine, 291 Campus Drive, Li Ka Shing Building, Stanford, CA 94305-5101, USA \\ ${ }^{2}$ Department of Pediatrics, Stanford Children's Hospital, 725 Welch Road, Palo Alto, CA 94304, USA \\ ${ }^{3}$ Division of Pulmonary Medicine, Department of Pediatrics, Stanford Children's Hospital, 725 Welch Road, Palo Alto, CA 94304, USA
}

Correspondence should be addressed to Jonathan D. Santoro; jsantoro@stanford.edu

Received 11 February 2015; Revised 26 March 2015; Accepted 2 April 2015

Academic Editor: Tomonobu Koizumi

Copyright (C) 2015 Lucy Schoen et al. This is an open access article distributed under the Creative Commons Attribution License, which permits unrestricted use, distribution, and reproduction in any medium, provided the original work is properly cited.

\begin{abstract}
Nocardia spp. are bacteria of low virulence that cause infection classically in immunocompromised hosts with the lungs as the primary site of infection in the majority of cases. Patients with cystic fibrosis have pulmonary disease characterized by frequent and progressive bacterial infections. Reports of Nocardia spp. isolation in CF are rare in the literature and may represent colonization or active infection, the significance and optimal treatment of which are unknown. We report the second case to date of Nocardia transvalensis pulmonary infection in an immunocompetent patient with CF and the first in a child under the age of eighteen.
\end{abstract}

\section{Background}

Nocardia spp. are gram positive, intracellular bacteria of low virulence found worldwide in soil and most frequently cause opportunistic infection in immunocompromised hosts. Incidence has been estimated at 1,000 cases annually in the United States, although rates are increasing [1]. Risk factors for nocardiosis include glucocorticoid use, IV drug use, previous transplant, acquired immune deficiency syndrome, and underlying pulmonary disease including chronic obstructive pulmonary disease, pulmonary fibrosis, and silicosis [2]. Immunocompetent hosts represent $10-50 \%$ of cases of infection [3]. Disease spectrum varies and includes pulmonary, neurologic, and/or cutaneous involvement. The lungs are the primary site of infection in two-thirds of cases, with radiographic findings ranging from small nodules to bilateral infiltrates with cavitation [3].

Cystic fibrosis (CF) is an autosomal recessive disease caused by mutations in a chloride ion channel resulting in multiple organ involvement with pulmonary manifestations as the major contributor to morbidity and mortality. Viscous secretions and chronic obstruction lead to colonization by bacteria and acute and chronic infection. Bacterial species influence progression of irreversible lung disease and greatly affect morbidity and mortality in this patient population despite their immunocompetence. Initial colonizers include Staphylococcus aureus and Haemophilus influenzae with Pseudomonas aeruginosa presenting later in the disease course [4]. Reports of isolating Nocardia spp. on sputum cultures in CF are rare in the United States, begging the question of whether the frequent and aggressive antimicrobial use in CF limits extensive growth of this microorganism [5-9].

The interface between CF and Nocardia spp. is fraught with difficulty. Nocardia spp. are slow growing and elusive on culture media. Further complicating assessment is a lack of understanding of this organisms' virulence and its role in the microbiota of the lung in a patient with CF. The majority of previously reported cases of Nocardia spp. appear to represent colonization of unknown clinical significance rather than active infection [5-8]. Pulmonary Nocardia spp. infection in CF has been reported rarely and, as such, the prognosis and optimal treatment are not well defined [7, 10-12]. Here we present a case of Nocardia transvalensis infection in an immunocompetent child with CF.

\section{Case Report}

A 9-year-old female with CF (homozygous Q890X mutation) was admitted from an outside hospital for fatigue, cough, and 
TABLE 1: Antimicrobial susceptibility charts for Pseudomonas aeruginosa and Staphylococcus aureus from previous hospitalization.

(a) Pseudomonas aeruginosa (nonmucoid CF)

\begin{tabular}{lrr}
\hline Antibiotic & & Sensitivity \\
\hline Amikacin & Method: $K B$ & Susceptible \\
Aztreonam & Method: $K B$ & Susceptible \\
Ceftazidime & Method: $K B$ & Susceptible \\
Ciprofloxacin & Method: $K B$ & Susceptible \\
Meropenem & Method: $K B$ & Susceptible \\
Piperacillin & Method: $K B$ & Susceptible \\
Ticarcillin & Method: $K B$ & Intermediate \\
Tobramycin & Method: $K B$ & Susceptible \\
\hline
\end{tabular}

(b) Staphylococcus aureus

\begin{tabular}{|c|c|c|}
\hline Antibiotic & Sensitivity & Microscan \\
\hline \multirow[t]{2}{*}{ Ciprofloxacin } & Resistant & $>2$ \\
\hline & Method: $M I C$ & \\
\hline \multirow[t]{2}{*}{ Clindamycin } & Resistant & $>4$ \\
\hline & Method: $M I C$ & \\
\hline \multirow[t]{2}{*}{ Erythromycin } & Resistant & $>4$ \\
\hline & Method: MIC & \\
\hline \multirow[t]{2}{*}{ Gentamicin } & Susceptible & $\leq 1$ \\
\hline & Method: MIC & \\
\hline \multirow[t]{2}{*}{ Levofloxacin } & Resistant & $>4$ \\
\hline & Method: $M I C$ & \\
\hline \multirow[t]{2}{*}{ Moxifloxacin } & Resistant & 4 \\
\hline & Method: $M I C$ & \\
\hline \multirow[t]{2}{*}{ Oxacillin } & Susceptible & \\
\hline & Method: MIC & \\
\hline \multirow[t]{2}{*}{ Penicillin } & Resistant & 2 \\
\hline & Method: $M I C$ & \\
\hline \multirow[t]{2}{*}{ Tetracycline } & Resistant & $>8$ \\
\hline & Method: MIC & \\
\hline \multirow[t]{2}{*}{ Trimeth-Sulfamethox } & Susceptible & $\leq 0.5 / 9.5$ \\
\hline & Method: $M I C$ & \\
\hline \multirow[t]{2}{*}{ Vancomycin } & Susceptible & 1 \\
\hline & Method: MIC & \\
\hline
\end{tabular}

fevers (Tmax $40^{\circ} \mathrm{C}$ ). At baseline her CF-related pulmonary disease was well controlled with a mean forced expiratory volume in one second (FEV1) of 93\% when being healthy. She had had recent hospitalization for CF exacerbation with sputum cultures positive for methicillin-resistant Staphylococcus aureus and Pseudomonas aeruginosa (Table 1). Past medical history was positive for pancreatic insufficiency and chronic
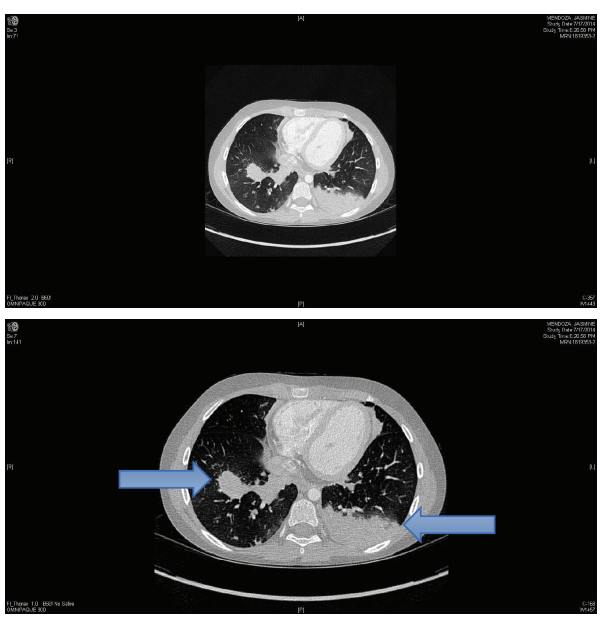

FIgure 1: Chest CT, axial view with multifocal pneumonia.

allergic bronchopulmonary aspergillosis (ABPA), which was well controlled after prednisolone course two months previously. She lived on a ranch with several domesticated animals but did not perform field work concerning Nocardia exposure.

On admission she was continued on ceftriaxone and her outpatient regimen including inhaled tobramycin, itraconazole, azithromycin, dornase alpha, acetylcysteine, and bronchodilators with vest treatments. Lab work including CBC, liver function tests, and chemistries was unremarkable and adjusted neutrophil count was within normal limits. On hospital day one she developed hypoxia and tachypnea requiring supplemental oxygen. Physical exam revealed a young girl in mild respiratory distress with reduced rightsided breath sounds and coarse crackles throughout the lung fields bilaterally without wheezes or stridor. She had a nonproductive cough and full inspiration was limited by right-sided flank pain. The remainder of her exam was unremarkable. Chest radiograph demonstrated a new right lower lobe consolidation. She was started on vancomycin, but despite broadened coverage she continued to be febrile to $40.4^{\circ} \mathrm{C}$ and complained of pleuritic chest pain. On hospital days one and two her oxygen requirements increased. Chest CT demonstrated a multifocal pneumonia (Figure 1). Initial sputum cultures returned positive for Nocardia species and blood cultures were negative. No other organisms were isolated from these cultures. Given continued symptoms despite broad antibiotic coverage and imaging results, the Nocardia spp. were considered to be active infection rather than colonization.

Antibiotic coverage was transitioned to IV sulfamethoxazole/trimethoprim ( $15 \mathrm{mg} / \mathrm{kg} /$ day $)$ and within 24 hours fevers resolved, respiratory status improved, and she was weaned off supplemental oxygen. Due to high rates of antibiotic resistance in some Nocardia species, double antibiotic coverage was proposed. IV linezolid (30 mg/kg/day) was initiated given favorable susceptibility profiles in Nocardia spp. Physical exam revealed no evidence of cutaneous involvement and brain MRI ruled out CNS nocardiosis. 
Speciation data returned Nocardia transvalensis with susceptibilities to amoxicillin-clavulanic acid, linezolid, sulfamethoxazole/trimethoprim, and resistance to amikacin. She tolerated 14 days of IV linezolid and 19 days of IV sulfamethoxazole/trimethoprim with slow improvement in energy and respiratory status. She had severe nausea in the first week of treatment leading to a weight loss of $3 \mathrm{~kg}(8 \%$ body weight). Repeat sputum cultures were negative for Nocardia spp. Pulmonary function was reduced compared to baseline with an FEV1 nadir of 50\%. She was discharged home on two weeks of oral sulfamethoxazole/trimethoprim and amoxicillin-clavulanic acid (30 mg/ $\mathrm{kg} /$ day) followed by monotherapy with oral sulfamethoxazole/trimethoprim for a three-month course. Sulfamethoxazole/trimethoprim dose was reduced to $11 \mathrm{mg} / \mathrm{kg} /$ day given nausea during hospitalization.

Repeat cultures at one month remained negative for Nocardia spp. and FEV1 was improved to $76 \%$. Chest radiograph at two months showed continued opacities and FEV1 remained below baseline at $85 \%$. She underwent bronchoscopy for persistent pulmonary findings and cultures were again negative for Nocardia spp. She completed three-month course of sulfamethoxazole/trimethoprim at which time her pulmonary function had returned to baseline with FEV1 of 95\%. Work-up for immunodeficiency was negative and chest $\mathrm{CT}$ at 6 months displayed no intrapulmonary changes.

\section{Discussion}

Pediatric patients with CF presenting with Nocardia spp. infection are rare but recent reports by Rodriguez-Nava et al. and Thorn et al. indicate a growing awareness that challenges prior data indicating Nocardia spp. as a pathogen of immunocompromised adults $[3,7,8]$. These larger scale studies present patients with both colonization and active infection, which can be difficult to differentiate given underlying lung pathology in CF and frequent growth of multiple pathogens on sputum culture causing debate with regard to clinical need to treat [7]. The authors of these studies advocate therapy when symptoms are present and Nocardia spp. are isolated alone or when symptoms persist in the context of multiple pathogens despite broad antibiotic coverage.

The standard treatment for pulmonary nocardiosis includes sulfamethoxazole/trimethoprim, although there have been reported cases of resistance [3, 7]. Treatment duration is usually 3-6 months but is not standardized and varies based on clinical course and comorbidities [3]. In CF patients with reported Nocardia spp. infections, antibiotic durations ranged from 15 days to over 6 months. The majority of patients received sulfamethoxazole/trimethoprim alone or in combination with other agents including amoxicillinclavulanic acid, clarithromycin, ciprofloxacin, ceftazidime, or amikacin [7, 10-12]. Therapy with linezolid alone has also been used [6]. The only prior report of Nocardia transvalensis infection in a patient with CF was successfully treated with two weeks of IV sulfamethoxazole/trimethoprim and linezolid followed by one week of oral linezolid and four weeks of oral sulfamethoxazole/trimethoprim [10].
Our case report presents the second report of Nocardia transvalensis in a patient with CF in the United States and the first in a child under 18. This subspecies of Nocardia has been previously identified as more virulent in mice than the more prevalent Nocardia asteroides subspecies [13]. Patients are more likely to develop greater symptom burden with this subspecies but general susceptibility patterns are favorable with common resistance only to amikacin noted at $18 \%$ [14]. Morbidity and mortality associated with this microorganism are generally limited to dissemination from the pulmonary system which is rare in immunocompetent patients. Further, assessment of dissemination can be nearly impossible to discern as growth on blood culture is almost universally negative [13]. Of note, there has been one case report in adult literature describing pulmonary nocardiosis with the presence of ABPA, which as with our patient may indicate need to treat [15]. Oral corticosteroids have been previously proposed as a risk factor for development of nocardiosis [3]; however, it is unknown whether ABPA or its therapy is a risk factor for Nocardial colonization or infection in CF.

The effect of Nocardia spp. and specifically Nocardia transvalensis colonization of the lung tissue is unknown, making it difficult to ascertain whether asymptomatic patients warrant antimicrobial therapy. Microbial burden in patients with CF has been studied previously, demonstrating need for eradication of certain microorganisms, but this has not been established for Nocardia spp. [16]. Previous studies have demonstrated an increased severity of symptoms with concurrent Nocardia spp. and Pseudomonas spp. infections, although this observation is strictly clinical in nature [17].

The microbiome of the CF patient's pulmonary system is diverse and fragile. While we advocate for antimicrobial stewardship, we believe that treatment of Nocardia transvalensis in patients with CF is necessary as both our patient and that of the previous reported case presented with active infection. The association between greater symptom burden and coinfection with Pseudomonas spp. in conjunction with the greater virulence of this Nocardia subspecies is enough to warrant therapy, especially in patients with CF who cannot easily tolerate pulmonary insults. Additionally based on a literature review and the results of our study and Aravantagi et al., we advocate for a three-month course of sulfamethoxazole/trimethoprim (initially by IV route then PO) in conjunction with IV linezolid during acute hospitalization [10].

This case is unique in both its rarity and complexity. We seek to highlight our case and recent literature in the setting of increased reports of Nocardia spp. colonization and infection in immunocompetent patients with CF over the last 20 years [18].

\section{Conclusion}

Our report seeks to stress the importance of antimicrobial therapy in patients with CF presenting with Nocardia transvalensis infection as the virulence of this organism appears to be greater than other Nocardia species in patients with CF. Finally we also would like to purpose a possible 
correlation between a diagnosis of ABPA in CF and developing Nocardia spp. infection.

\section{Consent}

Written informed consent was obtained from the patient for publication of this case report and any accompanying images.

\section{Conflict of Interests}

All authors deny any financial or nonfinancial conflict of interests in the production of this case report.

\section{Authors' Contribution}

Lucy Schoen and Jonathan D. Santoro contributed to the writing, literature review, and case management of the paper. Sumit Bhargava and Carlos Milla contributed to editorial review, literature review, and revisions of the paper. All authors approved the final version of this paper.

\section{References}

[1] B. L. Beaman, J. Burnside, B. Edwards, and W. Causey, "Nocardial infections in the United States, 1972-1974," The Journal of Infectious Diseases, vol. 134, no. 3, pp. 286-289, 1976.

[2] P. R. Georghiou and Z. M. Blacklock, "Infection with Nocardia species in Queensland: A review of 102 clinical isolates," Medical Journal of Australia, vol. 156, no. 10, pp. 692-697, 1992.

[3] E. R. Lederman and N. F. Crum, "A case series and focused review of nocardiosis: clinical and microbiologic aspects," Medicine, vol. 83, no. 5, pp. 300-313, 2004.

[4] R. L. Gibson, J. L. Burns, and B. W. Ramsey, "Pathophysiology and management of pulmonary infections in cystic fibrosis," American Journal of Respiratory and Critical Care Medicine, vol. 168, no. 8, pp. 918-951, 2003.

[5] M. I. Barrio, M. C. Martínez, C. Prados, R. M. Girón, L. Maiz, and M. T. Martínez, "Isolation of Nocardia species in patients with cystic fibrosis," Archivos de Bronconeumología, vol. 44, no. 2, pp. 109-112, 2008.

[6] R. Lumb, H. Greville, J. Martin, N. Sangster, and M. Holmes, "Nocardia asteroides isolated from three patients with cystic fibrosis," European Journal of Clinical Microbiology and Infectious Diseases, vol. 21, no. 3, pp. 230-233, 2002.

[7] V. Rodriguez-Nava, S. Durupt, and S. Chyderiotis, "A French multicentric study and review of pulmonary Nocardia spp. in cystic fibrosis patients," Medical Microbiology and Immunology, 2014.

[8] S. T. Thorn, M. A. Brown, J. J. Yanes et al., "Pulmonary nocardiosis in cystic fibrosis," Journal of Cystic Fibrosis, vol. 8, no. 5, pp. 316-320, 2009.

[9] C. Colombo and J. Littlewood, "The implementation of standards of care in Europe: state of the art," Journal of Cystic Fibrosis, vol. 10, supplement 2, pp. S7-S15, 2011.

[10] A. Aravantagi, K. Patra, M. Broussard, and K. Jones, "A case of Nocardia transvalensis pneumonia in a 19-year-old cystic fibrosis patient," Lung India, vol. 29, no. 3, pp. 283-285, 2012.

[11] Y. Pablo and T. Asher, "Pathological cases of the month," Archives of Pediatrics \& Adolescent Medicine, vol. 148, no. 2, pp. 209-210, 1994.
[12] F. Bittar, N. Stremler, J.-P. Audié et al., "Nocardia farcinica lung infection in a patient with cystic fibrosis: a case report," Journal of Medical Case Reports, vol. 4, article 84, 2010.

[13] H. C. Gugnani, I. C. Iboko, and S. E. Ikerionwu, "Pathogenicity of Nocardia transvalensis for laboratory mice," Mycopathologia, vol. 96, no. 2, pp. 79-86, 1986.

[14] M. M. McNeil, J. M. Brown, P. R. Georghiou, A. M. Allworth, and Z. M. Blacklock, "Infections due to Nocardia transvalensis: clinical spectrum and antimicrobial therapy," Clinical Infectious Diseases, vol. 15, no. 3, pp. 453-463, 1992.

[15] B. Sharma, G. Ghosh, U. Kamble et al., "Allergic broncho pulmonary aspergillosis complicated by nocardiosis," Case Reports in Pulmonology, vol. 2012, Article ID 758630, 5 pages, 2012.

[16] S. H. Pattison, G. B. Rogers, M. Crockard, J. S. Elborn, and M. M. Tunney, "Molecular detection of CF lung pathogens: current status and future potential," Journal of Cystic Fibrosis, vol. 12, no. 3, pp. 194-205, 2013.

[17] J. Beucher, C. Belleguic, G. Brinchault, E. Deneuville, P.-Y. Donnio, and M. Roussey, "Nocardia farcinica infection in a patient with cystic fibrosis," Revue des Maladies Respiratoires, vol. 27, no. 1, pp. 76-79, 2010.

[18] J. Ambrosioni, D. Lew, and J. Garbino, "Nocardiosis: updated clinical review and experience at a tertiary center," Infection, vol. 38, no. 2, pp. 89-97, 2010. 


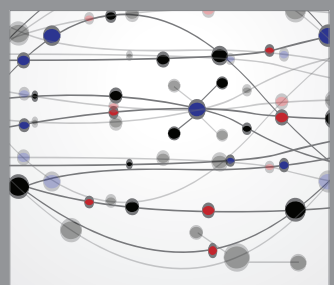

The Scientific World Journal
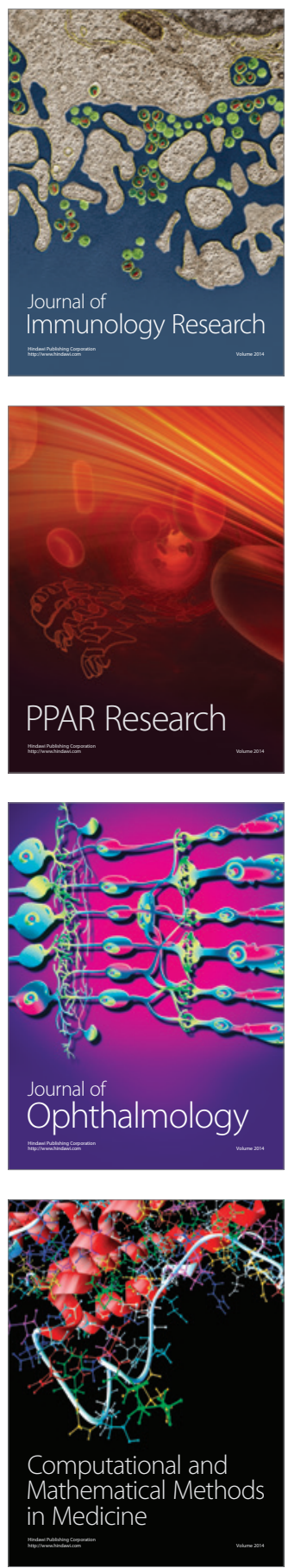

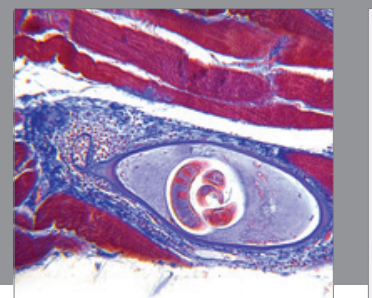

Gastroenterology

Research and Practice
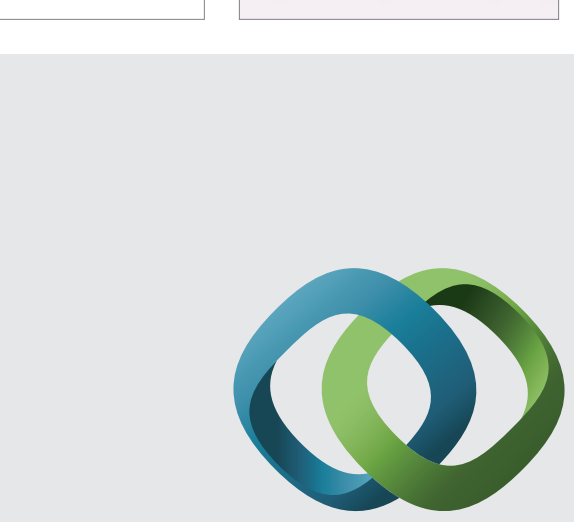

\section{Hindawi}

Submit your manuscripts at

http://www.hindawi.com
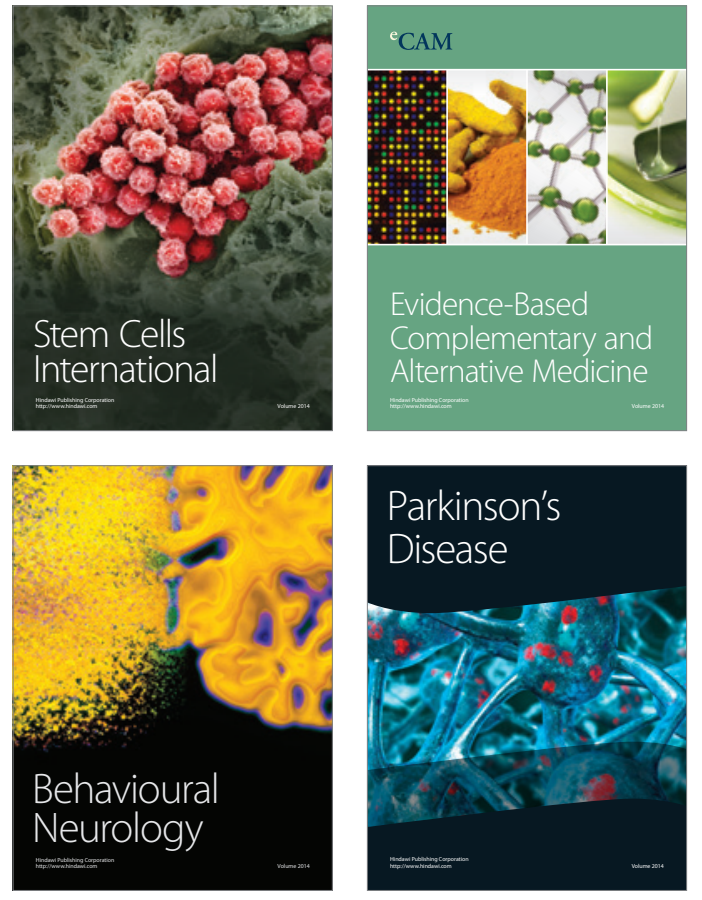
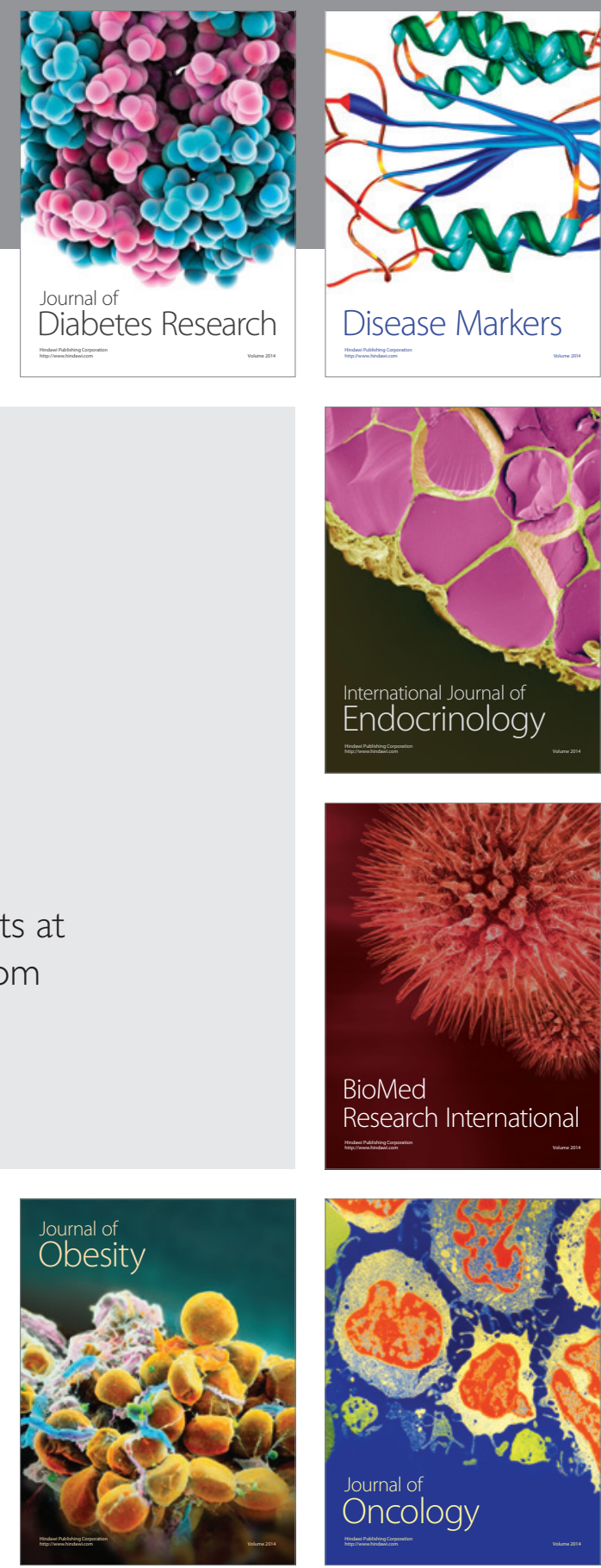

Disease Markers
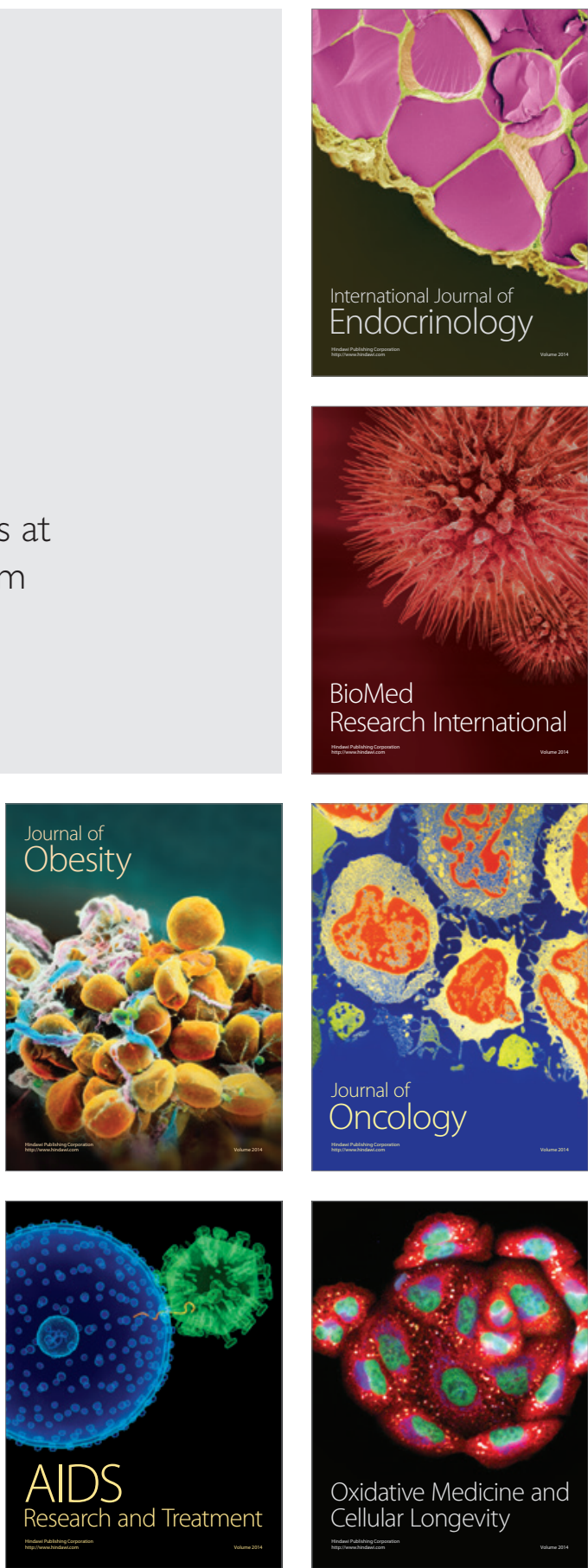